\title{
The effects of an exposure therapy training program for pre-professionals in an intensive exposure-based summer camp
}

\author{
Ryan J. McCarty ${ }^{1,2 *}$ (D), Danielle L. Cooke ${ }^{1,2 \#}$, Lacie M. Lazaroe ${ }^{1,2 \S}$, Andrew G. Guzick ${ }^{1,2 \ddagger}$, \\ Andrea D. Guastello ${ }^{1}$, Sierra M. Budd ${ }^{1}$, Seth T. Downing ${ }^{1,2}$, Ashley R. Ordway ${ }^{1}$, Carol A. Mathews ${ }^{1}$ \\ and Joseph P. H. McNamara ${ }^{1}$ \\ ${ }^{1}$ Department of Psychiatry, College of Medicine, University of Florida, 4197 NW 86th Terrace, Gainesville, FL 32606, USA \\ and ${ }^{2}$ Department of Clinical and Health Psychology, College of Public Health and Health Professions, University of Florida, \\ 1225 Center Drive, Gainesville, FL 32611, USA \\ *Corresponding author. Email: ryanmccarty12@ufl.edu
}

(Received 9 July 2021; accepted 23 November 2021)

\begin{abstract}
Although exposure therapy (ET) is an effective treatment for anxiety disorders and obsessive-compulsive disorder, many clinicians report not utilizing it. The present study targeted common utilization barriers by evaluating an intensive ET training experience in a relatively inexperienced sample of pre-professionals. Thirty-two individuals at the undergraduate or college graduate level without formal clinical experience participated as camp counsellors in a 5day exposure-based therapeutic summer camp for youth with anxiety disorders and/or obsessive-compulsive disorder. Participants were trained in ET through a progressive cascading model and answered questionnaires before and after camp. Repeated measure MANOVA revealed significantly increased feelings of self-efficacy conducting exposures, and significantly decreased feelings of disgust sensitivity and contamination-related disgust from pre-camp to post-camp. A subset of individuals providing data 1 month after the camp maintained a significant gain in ET selfefficacy. Regression analyses revealed that contamination-related disgust, but not disgust sensitivity, significantly predicted post-camp ET self-efficacy. These findings suggest that individuals early into their post-secondary education can learn ET, and the progressive cascading model holds promise in its utility across experience levels and warrants further investigation. Disgust may also play a role in feelings of competency conducting ET. Implications on dissemination and implementation efforts are also discussed.
\end{abstract}

\section{Key learning aims}

(1) How can training of CBT techniques such as exposure occur prior to graduate education?

(2) Can self-efficacy in conducting exposures meaningfully increase in an experiential training of preprofessionals?

(3) How does an individual's tolerance of disgust impact feelings of competence conducting exposures?

Keywords: CBT training; disgust; exposure therapy; self-efficacy

\footnotetext{
\#Present address: Department of Psychiatry (SOM), University of Colorado Anschutz Medical Campus, 13001 East 17th Pl, Aurora, CO 80045, USA

${ }^{\S}$ Present address: Rothman Center for Pediatric Neuropsychiatry, University of South Florida College of Medicine, 601 7th St S, St. Petersburg, FL 33701, USA

${ }^{\ddagger}$ Present address: Department of Psychiatry and Behavioral Sciences, Baylor College of Medicine, 1977 Butler Blvd, Houston, TX 77030, USA

(C) The Author(s), 2022. Published by Cambridge University Press on behalf of the British Association for Behavioural and Cognitive Psychotherapies. This is an Open Access article, distributed under the terms of the Creative Commons Attribution licence (https:// creativecommons.org/licenses/by/4.0/), which permits unrestricted re-use, distribution, and reproduction in any medium, provided the original work is properly cited.
} 


\section{Introduction}

Although exposure therapy (ET) is an effective treatment and a key element of cognitive behavioural therapy (CBT) for many anxiety disorders and obsessive-compulsive disorder (OCD; Abramowitz et al., 2019), a significant portion of mental health providers across the spectrum self-report that they either infrequently or never utilize ET in the treatment of anxiety for either youth (Higa-McMillan et al., 2017; Reid et al., 2018; Whiteside, Sattler et al., 2016) or adults (Becker et al., 2004; Freiheit et al., 2004; Hipol and Deacon, 2013; Pittig and Hoyer, 2017; van Minnen et al., 2010). Even among therapists who identify as cognitive behavioural therapists, use of ET is low compared with other cognitive behavioural techniques (Freiheit et al., 2004; Hipol and Deacon, 2013; Whiteside, Deacon et al., 2016) and when ET is utilized, clinicians report utilizing less effective forms of ET, such as imaginal exposures rather than in vivo exposures (Hipol and Deacon, 2013; Pittig and Hoyer, 2017; Sars and van Minnen, 2015; Reid et al., 2018).

Given this concern, determining the barriers to the implementation of ET has become of empirical interest in recent years. Many clinicians report concerns that use of ET will cause distress to the patient and exacerbate their symptoms (Becker et al., 2004; Deacon, Lickel et al., 2013; Zoellner et al., 2011), that patients will drop out of treatment (Deacon, Lickel et al., 2013; Olatunji et al., 2009), worry about legal liability due to exposures (Olatunji et al., 2009), and a belief that research findings do not translate to clinical practice (Gunter and Whittal, 2010; Olatunji et al., 2009). While these concerns are not supported by research (McGuire et al., 2018; Olatunji et al., 2009), endorsement of negative beliefs about ET has been shown to be associated with lower rates of self-reported usage (Meyer et al., 2014; Pittig et al., 2019; Reid, Bolshakova et al., 2017; Whiteside, Deacon et al., 2016), and cautious/ suboptimal exposure delivery in experimental and hypothetical situations (Deacon, Farrell et al., 2013; Farrell et al., 2013; Reid, Bolshakova et al., 2017), as well as in self-reported actual practice (Reid et al., 2018).

The personal characteristics of clinicians have also been identified as a barrier to practising ET. For instance, clinicians' own anxiety is associated with lower rates of self-reported ET use, less optimal exposure delivery, and larger endorsement of other barriers to ET (Harned et al., 2013; Levita et al., 2016; Meyer et al., 2014; Reid, Bolshakova et al., 2017; Scherr et al., 2015). Clinicians may experience anxiety during in vivo exposures (Levita et al., 2016; Schumacher et al., 2014) and some therapists worry that they will not be able to effectively tolerate their own distress during this time (Levita et al., 2016). As such, the emotion of disgust may be a particularly salient domain to investigate. Disgust appears to be an important clinical variable, as there is a large body of research linking high proneness to disgust with the development, maintenance and symptomatology of many forms of psychopathology, particularly certain phobias (e.g. blood, animal, health) and contamination fear (a common symptom of OCD; see Olatunji et al., 2017 for a review). Additionally, it has been posited that therapists' proneness to feelings of disgust may also play a role in the dissemination of ET when considering how frequently disgust is targeted in anxiety and OCD, as exposures may be avoided by clinicians as to not elicit this aversive emotion (Reid, Bolshakova et al., 2017; Reid, Guzick et al., 2017). As a result, therapists may inadvertently model avoidance behaviour through not conducting exposures or approaching exposures in an overly cautious nature (Reid, Bolshakova et al., 2017; Waller and Turner, 2016).

Clinicians have also reported a lack of formal training as a barrier to utilizing ET (Gunter and Whittal, 2010; Reid, Bolshakova et al., 2017; Reid et al., 2018; van Minnen et al., 2010). Indeed, individuals who report more extensive training in ET and identify as anxiety specialists are more likely to report utilizing ET in clinical practice (Becker et al., 2004; Hipol and Deacon, 2013; Sars and van Minnen, 2015; Scherr et al., 2015). Training interventions for ET in the form of online training or brief workshops have shown promise in decreasing negative attitudes toward ET, 
increasing knowledge, competence and confidence in conducting ET, and increasing self-reported rates of ET utilization (Deacon, Farrell et al., 2013; Farrell et al., 2013; Farrell et al., 2016; Harned et al., 2014; Jacoby et al., 2019; Reese et al., 2016). While most of these training sessions were open for clinicians of any level of experience (i.e. licensed providers or pre-doctoral trainees), only one was targeted toward novices (i.e. pre-professionals; Farrell et al., 2013). There is also concern that the effects of workshops on established providers do not persist over time (Beidas and Kendall, 2010; Chu et al., 2015); it is possible, therefore, that such training may be more effective for those earlier in their clinical training.

As such, there have been calls for more early clinical training in treatment modalities such as ET (Callahan and Watkins, 2018; Klepac et al., 2012). The progressive cascading model (PCM; Balkhi et al., 2016) is one of the few described methods of practicum training for novices in ET. In the PCM, ET is taught through a hierarchical structure and is based on a 'train-the-trainer' model, where individuals play different roles in the delivery of ET based on their comfort, understanding of principles, ability to utilize skills, autonomy, and ability to lead (Balkhi et al., 2016). In practice, individuals with limited experience are given lower levels of responsibility which gradually increase as training progresses. In addition, structured supervision is provided and tailored for trainees at each level. Often in traditional training, techniques such as exposures are considered 'advanced' techniques, and students do not practise these techniques until their later training (Balkhi et al., 2016), which is not ideal for maximizing ET dissemination. Rather, in the PCM, inexperienced individuals are provided early opportunities to build comfort and confidence with ET (e.g. acting as therapy aides or co-therapists) rather than either immediately throwing trainees into the 'deep end' or waiting long periods of time before trainees actively experience ET. Establishing early self-efficacy in one's ability to engage and conduct exposures is essential, as self-efficacy is perceived as an influential variable for therapists to adopt use of ET and evidence-based practices in general (Cook et al., 2009; Deacon, Farrell et al., 2013; Harned et al., 2013, 2014; Pittig et al., 2019).

The present study proposed to extend the PCM in a short-term but active, intensive and naturalistic training experience in ET via participation as camp counsellors in a therapeutic summer camp, 'Fear Facers'. Fear Facers is a 40-hour long, 5-day exposure-based therapeutic summer camp for individuals aged 7 to 16 years with a diagnosis of OCD and/or an anxiety disorder. Camp counsellors acted as 'exposure coaches' (see more below) and were, primarily, but not exclusively, undergraduate college students or recent graduates who had never conducted therapy or engaged in any graduate-level clinical practicum. This study aimed to assess whether these pre-professionals broadly interested in mental health and healthcare could build self-efficacy in conducting exposures following participation in this experience. Additionally, this study sought to examine the role of trainee's perceptions of disgust in this experience, through both its change from beginning to end of camp, as well as its relationship with ET self-efficacy. Disgust sensitivity has been previously demonstrated to decrease during graduate-level training in ET (Reid, Guzick et al., 2017), but it is unknown how disgust may influence ET self-efficacy. In the present study, a counsellor's own feelings of disgust were hypothesized to have a negative influence on ET self-efficacy, considering that the Fear Facers camp placed heavy emphasis on contamination and phobic-related fears (described below).

\section{Method}

\section{Procedure}

A retrospective review of training records of camp counsellors was conducted, as approved by a university ethical review board (University of Florida IRB201902582). Records of counsellors participating in the two summer 2018 and the two summer 2019 sessions of Fear Facers were obtained. Individuals were recruited to volunteer as counsellors for this program either 
through word-of-mouth or advertisements on a university web page for those interested in healthrelated professions. Counsellors completed in-person questionnaires before the training and after completing the week of camp to evaluate the success of the training program. One questionnaire was also administered by email one month following the end of camp. Some counsellors participated in multiple camp sessions; however, only their information from their first camp session with data was utilized. The trainees were reminded during data collection that all responses were de-identified. No compensation was provided.

\section{Participants}

Participants in this study consisted of 32 camp counsellors. Participants' ages ranged from 19 to 27 years (mean $=21.67, S D=2)$. The sample predominantly identified as female $(n=27,84.4 \%)$. Approximately two-thirds of the sample $(n=21,65.6 \%)$ self-reported that they had zero experience with ET. The remaining 11 participants reported having some informal experience with $\mathrm{ET}^{1}$ in a volunteer role, with years of experience averaging from 0.25 to 3 years. However, none of the participants had formal clinical experience. The majority of the sample $(n=18,56.3 \%)$ were undergraduate students in a 4 -year degree program, five $(15.6 \%)$ were recent bachelor's level graduates, and nine $(28.1 \%)$ were either first- or second-year graduate students in graduate health professions programs. All counsellors reported either being in or completing a psychology or pre-health undergraduate track. Additionally, all but two participants with previous ET experienced acquired their experience through a university clinic specializing in ET. Table 1 summarizes the demographic information described above.

\section{Training}

Before the beginning of the first day of a camp session, camp counsellors attended training lasting 2-4 hours that consisted of a mix of didactics and active practice in facilitating and conducting exposures in an optimal manner. Topics reviewed in this training include but were not limited to: the symptom presentation of OCD and anxiety, an explanation of how anxiety becomes impairing from a cognitive behavioural perspective, an explanation of the principles of exposure and response prevention, discussion of the theories positing exposure's mechanism of action, how to design exposures, how to encourage patients to engage in exposures, common myths regarding ET, and techniques to conduct exposures effectively. In addition, counsellors attended meetings prior to the start of camp to review information regarding the camper to whom they had been assigned, as well as to interact with the therapists assigned to the camper.

The clinical structure of camp follows the PCM, a hierarchically structured train-the-trainer model which is described extensively in Balkhi et al. (2016). At all times, at least one licensed psychologist was on-site and available to provide real-time supervision. Each camper was assigned a treatment team consisting of two therapists, typically a combination of a more experienced and less experienced graduate practicum student in psychology or mental health counselling, and a camp counsellor. Camp counsellors served as therapy aides during individual therapy sessions, and throughout the rest of the day served as exposure 'coaches' through facilitating the completion of in-camp exposure challenges established during therapy sessions, actively participating in camp activities, and modelling approach and non-avoidant behaviours. Although counsellors were assigned to specific campers, many camp activities involved splitting up counsellors and campers into various groups; thus, counsellors had opportunities to work with several campers. Counsellors were trained to readily seek supervision or assistance at any point in time they felt it was needed. Additionally, supervisors

\footnotetext{
${ }^{1}$ Participants were asked to estimate the amount of time they had been volunteering in a setting that utilized exposure therapy. Most participants with experience had previously volunteered with a university psychology clinic specializing in ET.
} 
Table 1. Sample characteristics $(n=32)$

\begin{tabular}{lrrlrr}
\hline Measure & $\%$ & $n$ & Measure & $\%$ & $n$ \\
\hline $\begin{array}{l}\text { Gender identification } \\
\quad \text { Female }\end{array}$ & & & Educational status & & \\
$\quad$ Male & 84.4 & 27 & 2nd year undergraduate & 12.5 & 4 \\
Racial/ethnic identification & 15.6 & 5 & 3rd year undergraduate & 9.4 & 3 \\
$\quad$ Asian & 15.6 & 5 & 4th year undergraduate & 34.4 & 11 \\
$\quad$ Arab/Middle Eastern & 3.1 & 1 & Post-baccalaureate & 15.6 & 5 \\
$\quad$ Latino or Hispanic & 37.5 & 12 & 2nd year graduate & 15.6 & 5 \\
$\quad$ White (non-Hispanic) & 43.8 & 14 & Session of camp & 12.5 & 4 \\
Prior ET experience & & & June 2018 & 56.2 & 18 \\
$\quad$ No & 65.6 & 21 & July 2018 & 21.9 & 7 \\
$\quad$ Yes & 34.4 & 11 & June 2019 & 12.5 & 4.4 \\
Less than 1 year & & 1 & July 2019 & 3 \\
1-2 years & & 5 & & & \\
2-3 years & & 5 & & \\
\hline
\end{tabular}

Prior ET experience was chiefly experience participants had as volunteers within an ET clinic. Undergraduate and graduate majors all fell within the health professions umbrella, most frequently medicine and psychology, as well as some nursing students. Information regarding the exact major for each participant was not available.

and more advanced therapists regularly checked in with counsellors and their patients, through group therapy, camp activities, or individual encounters.

Each camp day consisted of camper participation in an hour-long individual therapy session, a group therapy session, and a variety of camp games and activities. Camp activities included team building games, as well as artistic, sport, and other performance-related activities and games with creative twists designed to challenge common fears and symptom domains, such as contamination, perfectionism, social anxiety, and phobias. These activities incorporated exposure and response prevention exercises that encouraged campers to 'face their fears' in a variety of contexts. Some examples of this include playing 'hot potato' with a 'contaminated' object, conducting scavenger hunts with exposure goals, playing games in an incorrect or uneven manner, presenting in front of others about odd topics, and participating in a bug zoo demonstration.

\section{Measures}

\section{Confidence in Conducting Exposures Scale (CCES)}

The CCES (see Supplement 1 of the Supplementary material) was developed by the personnel in charge of training at the Fear Facers camp to assess volunteers' self-efficacy in conducting ET. The CCES is a self-report scale consisting of nine items. Each item asks participants to rate how confident they currently feel about conducting elements of ET on a 0 to 100 scale, where $0=$ 'cannot do at all', $50=$ 'moderately certain can do' and $100=$ 'highly certain can do'. These elements were derived from previous research investigating the training of ET (e.g. Harned et al., 2011). Example items include 'design an exposure for a patient', 'challenge a patient to participate in an exposure' and 'conduct an exposure independently'. A total score was created by averaging all nine items, and the internal consistency for the present sample was found to be excellent (Cronbach's $\alpha=0.98$ ). This measure was administered at pre-camp, post-camp, and 1 month following the end of camp.

\section{Disgust Scale-Revised (DS-R)}

The DS-R (Olatunji et al., 2007) is a psychometrically validated and widely used self-report scale assessing disgust sensitivity in multiple contexts. Disgust sensitivity has been defined as the propensity to experience disgust as aversive (Olatunji et al., 2007). The DS-R contains 25 items assessed on a 0 to 4 Likert scale. A total average score was used in the present study, 
with higher scores indicative of higher sensitivity to disgust. Internal consistency was found to be acceptable (Cronbach's $\alpha=0.78$ ). This measure was administered to the counsellors at pre-camp and post-camp.

\section{Contamination Disgust Scale (CDS)}

A modified ${ }^{2}$ version of the Contamination Cognition Scale (Deacon and Olatunji, 2007), labelled as the CDS in the present study (see Supplement 2 of the Supplementary material), was utilized to assess participants' estimation of the disgust they would experience if they touched a potentially contaminated item and could not wash their hands. The scale includes 13 items that patients with OCD often associate with germs, including toilet seats, raw meat, and animals. Participants rated their disgust on a 0 to 100 scale, where $0=$ 'not at all disgusted', $50=$ 'moderately disgusted' and $100=$ 'extremely disgusted'. A total score averaging all 13 items was calculated, and the scale demonstrated good internal consistency (Cronbach's $\alpha=0.92$ ). This measure was administered pre-camp and post-camp.

\section{Statistical Analyses}

Data were analysed using the program IBM SPSS 25. Preliminary preparation of the data revealed that $9.9 \%$ of all potential pre- and post-camp data points were missing, with Little's Missing Completely at Random analysis suggesting the data was missing at random $\left(\chi^{2}(30)=26.35\right.$, $p=0.657)$. Multiple imputation with 25 imputations was conducted to impute for missing values of the variables of interest at pre-camp and post-camp, using those variables along with all demographic variables as predictors. Pooled statistics across all 25 imputations are reported when possible, otherwise ranges of statistics across all imputations are presented.

As a preliminary analysis, a multivariate analysis of variance (MANOVA) was conducted to determine whether there was an association between particular demographic variables (i.e. prior volunteer experience with ET, level of education, and session of camp) and the pre- and post-camp variables of interest (i.e. CCES, DS-R and CDS scores). Prior experience with ET was dichotomized as yes (1) or no (0), while level of education was divided into three groups: current undergraduate students, post-baccalaureates, and current graduate students.

A repeated measures multivariate analysis of variance (RM-MANOVA) was then conducted to investigate changes in ET self-efficacy and disgust sensitivities from pre- to post-camp. CCES scores, DS-R scores and CDS scores were entered as dependent variables, and time of assessment (pre-camp or post-camp) was entered as the within-subjects factor. Additionally, the interactions between time and the demographic variables found to have a significant multivariate effect from the preliminary MANOVA were also evaluated to account for any confounding relationships. For effect sizes, $\eta^{2}$ partial was calculated for multivariate and univariate effects, while Cohen's $d$ for either between or within subjects was calculated for pairwise comparisons.

A separate RM-ANOVA was also conducted for participants who provided CCES data at pre-, post- and follow-up assessment. Imputed variables were not used for this analysis, as only 14 of the 32 participants provided these data. Bonferroni corrections were applied to these analyses to correct for multiple comparisons.

Lastly, two linear regressions were conducted to test whether ET self-efficacy was related to the disgust variables at either pre-camp or post-camp. The first predicted pre-camp CCES score using pre-camp DS-R and CDS scores, as well as any significant demographic variables. The second was conducted with pre-camp CCES score, post-camp DS-R and CDS scores, and significant demographic variables predicting post-camp CCES score.

\footnotetext{
${ }^{2}$ The original measure asked participants to rate how likely it was that they would become contaminated from touching an object, as well as how bad it would it be if it was actually contaminated. In the present study, participants were asked how much disgust they would experience if they touched the object and were unable to wash their hands afterwards.
} 
Table 2. Model statistics of RM-MANOVA tests

\begin{tabular}{lcccc}
\hline Effects and variables & $F$ range & d.f. & $p$ range & $\eta^{2}$ partial range \\
\hline Multivariate effect of time & $46.54-52.84$ & 3,28 & $<.001$ & $0.83-0.85$ \\
Confidence conducting exposures & $112.69-121.18$ & 1,30 & $<.001$ & $0.79-0.80$ \\
Disgust sensitivity & $19.58-24.26$ & 1,30 & $<.001$ & $0.40-0.45$ \\
Contamination disgust & $17.89-19.58$ & 1,30 & $<.001$ & $0.37-0.40$ \\
Multivariate effect of prior ET experience & $7.78-8.20$ & 3,28 & $<.001$ & $0.46-0.47$ \\
Confidence conducting exposures & $16.22-16.83$ & 1,30 & $<.001$ & $0.35-0.36$ \\
Disgust sensitivity & $0.55-0.97$ & 1,30 & $.333-.463$ & $0.02-0.03$ \\
Contamination disgust & $8.16-8.70$ & 1,30 & $.006-.008$ & $0.21-0.23$ \\
Multivariate interaction of time and prior ET experience & $10.17-10.94$ & 3,28 & $<.001$ & $0.52-0.54$ \\
Confidence conducting exposures & $28.26-31.09$ & 1,30 & $<.001$ & $0.49-0.51$ \\
Disgust sensitivity & $0.00-0.14$ & 1,30 & $.708-.991$ & $>0.01$ \\
Contamination disgust & $3.743-4.27$ & 1,30 & $.048-.063$ & $0.11-0.13$ \\
\hline
\end{tabular}

Based on 25 imputations. Time and timexprior ET experience entered as within subjects' factors; prior ET experience entered as between subjects' factor Pillai's trace for each multivariate effect is equivalent to its $\eta^{2}$ partial value.

Table 3. Estimated marginal means

\begin{tabular}{lccc}
\hline Time point and variable & $\begin{array}{c}\text { Prior ET experience } \\
(n=11)\end{array}$ & $\begin{array}{c}\text { No prior ET experience } \\
(n=21)\end{array}$ & $\begin{array}{c}\text { Total sample } \\
(n=32)\end{array}$ \\
\hline Pre-camp confidence conducting exposures & $70.96(6.70)^{\mathrm{a}, \mathrm{b}}$ & $30.27(4.85)^{\mathrm{b}, \mathrm{c}}$ & $50.61(4.14)^{\mathrm{d}}$ \\
Post-camp confidence conducting exposures & $89.09(3.13)^{\mathrm{a}}$ & $85.13(2.27)^{\mathrm{c}}$ & $87.11(1.93)^{\mathrm{d}}$ \\
Pre-camp disgust sensitivity & $41.27(4.17)^{\mathrm{e}}$ & $45.39(3.05)^{\mathrm{f}}$ & $43.33(2.58)^{\mathrm{g}}$ \\
Post-camp disgust sensitivity & $30.09(3.71)^{\mathrm{e}}$ & $33.35(2.71)^{\mathrm{f}}$ & $31.72(2.29)^{\mathrm{g}}$ \\
Pre-camp contamination disgust & $18.11(4.91)^{\mathrm{h}}$ & $35.84(3.56)^{\mathrm{h}, \mathrm{i}}$ & $26.98(3.03)^{\mathrm{j}}$ \\
Post-camp contamination disgust & $11.05(3.02)$ & $16.52(2.19)^{\mathrm{i}}$ & $13.78(1.86)^{\mathrm{j}}$ \\
\hline
\end{tabular}

Pooled mean (SE) statistics based on 25 imputations. All variables can range from 0 to 100 . Variables with the same superscript letter are significantly different from each other at $p<.05$.

\section{Results}

\section{Preliminary analyses}

The first MANOVA revealed a significant multivariate effect for prior ET experience on the precamp and post-camp variables across all 25 imputations (Pillai's trace $\geq .596, F_{6,20} \geq 4.908$, $p \leq .003$ ). Level of educational attainment (Pillai's trace $\leq .493, F_{12,42} \leq 1.112, p \geq .376$ ) and session of camp (Pillai's trace $\leq .482, \quad F_{18,66} \leq 0.722, \quad p \geq .777$ ) did not have significant multivariate effects across all imputations. As such, the subsequent RM-MANOVA was conducted using the interaction between time and prior ET experience, and prior ET experience was included as a predictor for both sets of regressions predicting CCES scores.

\section{Change in variables}

The RM-MANOVA revealed a significant main effect of time on the dependent variables, collectively, for all 25 imputations (all $p<.001$ ), with $\eta^{2}$ partial values ranging from 0.83 to 0.85 . Additionally, there was a significant multivariate interaction between time and prior ET experience for all 25 imputations (all $p<.001$ ), with $\eta^{2}$ partial values ranging from 0.52 to 0.54 . Multivariate and univariate effects are summarized in Table 2 and estimated marginal means for each variable at each time point are presented in Table 3.

\section{Self-efficacy conducting ET following training}

Both the main effect of time $\left(F_{1,30} \geq 112.69, p<.001, \eta_{\text {partial }}^{2} \geq 0.79\right)$ and the interaction of time and prior ET experience $\left(F_{1,30} \geq 10.17, p<.001, \eta_{\text {partial }}^{2} \geq 0.49\right)$ were significant for CCES scores across 
all imputations. Overall, ET self-efficacy increased by 36.5 points $(72 \%$ increase, $p<.001$ for all imputations) from pre-camp to post-camp, corresponding to a pooled Cohen's $d$ of 1.86. Across all imputations, pairwise comparisons revealed significant increases in ET self-efficacy for both those with prior experience $(d=1.19, p<.005)$ and those without prior experience $(d=2.92$, $p<.001)$ from pre-camp to post-camp. Those who had prior ET experience also reported significantly higher pre-camp ET self-efficacy than those without $(d=1.23, p<.001)$; however, there was no significant difference between the two groups in post-camp ET selfefficacy $(d=0.27, p>0.3)$.

The RM-ANOVA for the participants ${ }^{3}$ providing pre-, post- and follow-up data revealed a statistically significant effect of time $\left(F_{1.13,15.81}=26.22, p<.001, \eta_{\text {partial }}^{2}=0.70\right)$. Pairwise comparisons revealed the following differences in ET self-efficacy at each time point; postcamp was significantly higher than at pre-camp $(d=1.42, p<.001)$, post-camp was significantly higher than at follow-up $(d=0.84, p=.023)$, and follow-up was significantly higher than at pre-camp $(d=1.43, p<.001)$. Table 4 summarizes these findings.

\section{Disgust sensitivity following training}

The univariate effect of time was significant on DS-R scores for all 25 imputations $\left(F_{1,30} \geq 19.58\right.$, $p<.001, \eta_{\text {partial }}^{2} \geq 0.40$ ), but the interaction between time and prior experience was not $\left(F_{1,30} \geq 0.00, p \geq .708, \eta_{\text {partial }}^{2} \geq 0.00\right]$. Pairwise comparisons revealed that on average across all imputations, mean disgust sensitivity decreased by 11.61 points $(27 \%$ decrease, $p<.001$ for all imputations) from pre-camp to post-camp, corresponding to a pooled Cohen's $d$ of 0.84 . Disgust sensitivity did not significantly differ between those with and without prior ET experience at either time point.

\section{Contamination disgust following training}

The main effect of time for CDS scores was significant across all 25 imputations $\left(F_{1,30} \geq 17.89\right.$, $p<.001, \eta^{2}$ partial $\left.\geq 0.37\right)$. The interaction between time and prior ET experience on CDS scores reached significance at $\alpha=.05$ for two of the 25 imputations but approached significance in all other imputations $\left(F_{1,30} \geq 3.743, p\right.$ ranges .048 to $.063, \eta^{2}$ partial $\left.\geq 0.11\right)$. Across all participants, the pooled decrease in contamination disgust was 13.2 points $(49 \%$ decrease, $d=0.80$, $p<.001$ for all imputations). For those without prior experience with ET, contamination disgust significantly decreased ( $p<.001$ for all imputations) with a pooled Cohen's $d$ of 1.17. There was not a significant decrease in contamination disgust for those with prior ET experience (pooled $d=0.43, p<.16$ for all imputations). While those without prior ET experience reported higher contamination disgust at pre-camp (pooled $d=1.09, p<.001$ for all imputations), there was no significant difference between the two groups at post-camp (pooled $d=0.55, p<.15$ for all imputations).

\section{Predictors of ET self-efficacy}

Table 5 summarizes the regressions predicting pre-camp and post-camp ET self-efficacy. The imputed models for the first regression explained $46-47 \%$ of the variance in pre-camp ET self-efficacy $\left(F_{3,28} \geq 7.90, p<.001\right)$. In this first model, having prior experience with ET was a significant positive predictor of ET self-efficacy (pooled $\beta=0.72$, pooled $p<.001$ ), while precamp disgust sensitivity (pooled $\beta=-0.10$, pooled $p=0.542$ ), and pre-camp contamination disgust (pooled $\beta=0.14$, pooled $p=0.425$ ) were not.

\footnotetext{
${ }^{3}$ These 14 participants had pre-camp CCES scores that trended towards being significantly higher than the participants who did not complete the follow-up measure. No significant difference was found for post-camp CCES scores. Six of the 14 participants had prior ET experience.
} 
Table 4. Confidence conducting exposures at three time points

\begin{tabular}{lccc}
\hline & Pre-camp Mean (SD) & Post-camp Mean (SD) & Follow-up Mean (SD) \\
\hline Confidence conducting exposures & $55.60(30.36)$ & $88.89(8.45)$ & $79.96(16.42)$ \\
\hline
\end{tabular}

$n=14$, all significantly differ from each other at $p<.05$. Of these participants, six had prior ET experience.

Table 5. Regression statistics

\begin{tabular}{lcrrrr}
\hline Variable & $R^{2}$ range & $F$ range & $p$ & $t$ & $\beta$ \\
\hline Pre-camp confidence conducting exposures & $0.46-0.47$ & $7.90-8.24$ & $\leq 0.001$ & \\
$\quad$ & & 0.000 & 4.56 & 0.72 \\
Prior ET experience & & & -0.542 & -0.10 \\
Pre-camp disgust sensitivity & & 0.425 & 0.80 & 0.14 \\
Pre-camp contamination disgust & $0.50-0.54$ & $6.87-8.05$ & $\leq 0.001$ & & \\
Post-camp confidence conducting exposures & & & 0.027 & -2.21 & -0.40 \\
Prior ET experience & & & 0.000 & 3.84 & 0.72 \\
Pre-camp confidence conducting exposures & & & 0.292 & 1.05 & 0.17 \\
Post-camp disgust sensitivity & & 0.003 & -2.97 & -0.47 \\
Post-camp contamination disgust & & & & & \\
\hline
\end{tabular}

$R^{2}$ and $F$ values are the minimum and maximum statistics observed for the 25 imputations. $\beta$ and $t$ are pooled statistics of the 25 imputations.

In the second regression, between 50 and $54 \%$ of the variance in post-camp ET self-efficacy was explained across 25 imputations $(F 4,27 \geq 6.87, p<.001)$. Pre-camp ET self-efficacy was a significant positive predictor of post-camp ET self-efficacy (pooled $\beta=0.72$, pooled $p<.001$ ). Additionally, having prior experience with ET before participating in the camp and post-camp contamination disgust were both significant negative predictors of post-camp ET self-efficacy (pooled $\beta=-0.40$, pooled $p=.027$, and pooled $\beta=-0.47$, pooled $p=.003$, respectively). Postcamp disgust sensitivity was not a significant predictor in the model (pooled $\beta=0.17$, pooled $p=.292$ ).

\section{Discussion}

Consistent with hypotheses, participants' feelings of self-efficacy conducting ET significantly increased between the beginning of the training and the end of the summer camp, with a large effect size. This increase in self-efficacy was especially pronounced in individuals who reported they had no experience conducting exposures prior to the camp. Additionally, among a subset of participants surveyed one month following the end of the camp, average self-efficacy with ET remained above levels observed before the camp, although below levels observed immediately after the end of camp. Taken together, these findings contribute to research suggesting that brief training experiences that emphasize experiential learning, tiered supervision, and a train-the-trainer model can increase feelings of self-efficacy in performing ET or other forms of evidence-based practice, which has been linked with an increased likelihood of using the treatment (Harned et al., 2011; Harned et al., 2014; Pittig et al., 2019). These previous investigations, however, have utilized samples of currently practising clinicians, whereas the present study investigated individuals who had never conducted therapy or independent clinical work. There are several advantages to targeting this population.

First, providing early opportunities for individuals to gain experience conducting ET is associated with an increased likelihood of utilizing this treatment in the future (Broicher et al., 2017; Pittig and Hoyer, 2017). In general, clinicians directly report that early training experiences are highly influential in their decision to utilize certain psychotherapeutic practices (Cook et al., 2009). Additionally, younger, more inexperienced individuals are more open to undergo training in evidence-based modalities and ultimately adopt the treatment 
(Beidas and Kendall, 2010; Pittig and Hoyer, 2017). Thus, from a developmental lens, early experiences - particularly experiences prior to graduate-level training - with empirically supported treatments such as ET can be important in establishing a professional identity in which evidence-based practices are viewed as favourable and feasible to conduct in actual practice. Drawing from vocational theory, experiences like these can serve as opportunities to build interest in career choice through development of career self-efficacy (Choi et al., 2012). Specifically, this kind of training serves as a method to affect both process career self-efficacy (which refers to actively engaging in activities to make decisions on a career) and content career self-efficacy (referring to self-efficacy performing in certain fields, like exposure therapy in this case; Choi et al., 2012).

An additional advantage to this population is that it can potentially result in interdisciplinary dissemination of ET. Providing hands-on training in how to combat anxiety for those who may enter medicine, nursing or other health fields can be valuable in enhancing their treatment conceptualization and recommendations moving forward (Reid, Guzick et al., 2017). Calls to disseminate ET to lay-providers or non-mental health specialists have already been made, and findings suggest it can be effectively taught (Gega et al., 2007; McDonough and Marks, 2002).

In examining the CCES scores, one may be surprised by the high average level of ET selfefficacy following the end of camp (86.5 out of a possible 100) for a group of relative novices. Certainly, there is evidence to suggest that providers may over-estimate their own capabilities or competence with psychotherapeutic treatments (Waller and Turner, 2016). There is also the possibility of a ceiling effect of the CCES measure. However, it may also be attributable to the extensive clinical support provided to the counsellors through the PCM. Research has previously described the important role of organizational and environmental factors on feelings of self-efficacy conducting evidence-based practice (Beidas and Kendall, 2010; Turner et al., 2011). Through the PCM, counsellors were able to take limited responsibility and heavily lean on experienced therapists or supervisors, yet were also given numerous opportunities to directly experience and even administer the delivery of exposures, which was associated with greatly enhanced self-efficacy with ET even one month following camp. With that said, this increase in self-efficacy is heavily contingent on contextual factors, as participants' rating of self-efficacy is a function of their specific role in camp, so it is unknown if this would generalize to exposure therapy in other contexts.

Even amongst pre-professionals, those who had some level of 'experience' with ET came into the training experience with much higher feelings of exposure self-efficacy. Rather than exclude these individuals, these counsellors were included in the analyses because the PCM is designed to meet trainees where they are at in terms of their own capabilities (Balkhi et al., 2016), which is in line with conceptualizations that training should become increasingly complex as individuals progress (Callahan and Watkins, 2018). Counsellors entering with some experience were often assigned campers with more severe symptoms and more complex presentations or were given more responsibilities in setting up or leading camp-related activities. This may be reflective in that, while ET self-efficacy did increase amongst those with prior experience, post-camp selfefficacy did not significantly differ from those without prior experience. More experienced individuals may have had a different conceptualization as to their role in delivering ET, reflective of a developmentally tailored experience.

Both counsellor global disgust sensitivity and contamination disgust decreased with time, although the decrease in contamination disgust was only significant in those without prior ET experience. This is probably due to a combination of a floor effect on the CDS - those with prior ET experience already scored fairly low on this measure - and constraints related to the small sample size for the prior ET experience group $(n=11)$. Disgust sensitivity was previously observed to decrease across a practicum experience in ET following the PCM (Reid, Guzick et al., 2017), and has also been shown to reduce during ET for individuals independently of their anxiety (Smits et al., 2002). Of note, declines in disgust following 
exposure tend to be situation specific as opposed to global (Consedine et al., 2013; Olatunji et al., 2017; Smits et al., 2002), so perhaps these changes in disgust are contextualized to the setting of the camp. This may have been accomplished by the opportunities that counsellors had to model disgust-related exposures, as well as modelling they witnessed from more advanced trainees.

Moreover, in this study disgust sensitivity broadly did not predict ET self-efficacy; however, lower contamination disgust after camp was associated with higher post-camp ET self-efficacy. This may be because the camp's group activities tended to target contamination, phobic, and social fears, rather than obsessions and compulsions related to harm, symmetry or taboo thoughts. For example, counsellors and campers were instructed to not wash their hands while at camp except under specific circumstances, and many activities were designed to elicit feelings of being potentially contaminated. Based on their camp experience, pre-professionals in this study may have been more inclined to think that exposures typically involve contamination and may have been more likely to believe there is a stronger relationship between ability to deliver ET with how disgusted they feel with possibly contaminated objects as opposed to more broad disgust sensitivity. On the other hand, it is possible that specific feelings of disgust are more pertinent to beliefs of being able to effectively conduct ET than a more general disgust sensitivity. A study by Holstermann et al. (2009) found that feelings of task-specific disgust moderated changes in perceived self-efficacy related to that task. Like this study, disgust did not seem to impact self-efficacy prior to the start of the task but did impact participants' ratings of self-efficacy after the task had begun (Holstermann et al., 2009). Results from Randler et al. (2013) also suggest that situation-specific disgust may negatively impact feelings of competence.

This possible relationship between disgust and feelings of self-efficacy appears to be consistent with other research regarding disgust's impact on vocational development. Disgust sensitivity has been found to be associated with certain vocational decisions, such as choosing medicine over other healthcare professions (Consedine et al., 2013), or being more willing to engage in direct volunteer activities over indirect participation (Hamerman and Schneider, 2018). Randler and colleagues (2013) and Holstermann et al. (2009) suggested that feelings of disgust relating to academic activities may negatively influence intrinsic motivation, which may also apply in a context such as ET. Notably, average levels of disgust sensitivity and contamination disgust in this study were below the midpoint of the DS-R and CDS, respectively, at pre-camp and post-camp, thus, it is possible that individuals interested in participating in ET may present with lower or more easily adjusted state or trait disgust, but the present study cannot provide explicit support for that hypothesis.

In considering these findings, it is also important to note the limitations of the present investigation. This study did not have a control group and was not experimental in nature, thus statements regarding the 'effect' of this training must be tempered. That stated, this study possesses ecological validity due to the examination of an actual clinical event (i.e. clinical care during the Fear Facers camp) and the scalability and replicability of the PCM due to its focus on experiential learning with a hierarchical structure with tiered supervision (Balkhi et al., 2016). However, the sample size for the present study was limited, there was heterogeneity in participants' education levels and majors, and there was certainly selection bias due to use of a convenience sample. Additionally, two of the three main outcome measures were created by the authors for the purpose of this training, and thus have unestablished indices of reliability and validity. Other important measures of training outcome and impact such as satisfaction with utilizing ET, desire to utilize ET in the future, and directive measures of ET knowledge and competence were not included, nor was extensive longitudinal data gathered. As was observed, ET self-efficacy at 1-month follow-up decreased relative to immediately after camp, so it is unknown whether the gain in ET self-efficacy observed would remain over a longer period of time. It is also important to note that while feelings of competence utilizing a therapy are often associated with utilization rates, this is far from a perfect relationship 
(Beidas and Kendall, 2010). In this training experience, individuals were only assessed following the initial didactic training and after the end of camp, but not following the end of didactic training. As such, the relative effect of the pre-camp training versus the actual camp experience on the variables of interest is unknown. The information that was collected was not linked to patient outcomes, which can be problematic given that change in therapist variables such as self-efficacy have not always corresponded with patient change (Beidas and Kendall, 2010), although it is important to note the preliminary clinical data for patient outcome in Fear Facers is promising (L. Lazaroe et al., under review).

Future research should include experimental research utilizing larger, diverse samples, psychometrically validated questionnaires, and longitudinal data collection to verify the efficacy of the PCM, both in terms of trainee development as well as patient satisfaction and outcome. Use of control interventions (e.g. didactic instructions without hands-on experience) would also increase confidence in the efficacy of this training model. Additionally, more research is needed to directly identify the benefits of receiving early hands-on experience with evidence-based practices like ET, such as its role in vocational selection. The role of clinicians' feelings of disgust surrounding exposures warrant further investigation, as it is unknown if disgust is influential for more experienced clinicians. Clinician characteristics, particularly disgust proneness, should be holistically conceptualized and considered for their possible influence in treatment outcome.

\section{Conclusions}

In conclusion, a time-limited training experience that follows the principles of a live tieredsupervision model with modelling from more advanced clinicians and a hierarchical structure that provides incrementally more responsibility with increased competence is a promising method to enhance feelings of self-efficacy conducting ET, even amongst a group of preprofessionals with limited ET experience. In turn, there is potential that establishing early selfefficacy in ET may lead to increased dissemination of this efficacious treatment. While more research is needed on how therapist characteristics influence utilization of evidence-based practice, particularly disgust, we hope that these findings help guide future dissemination work and training efforts, with the goal of increasing the availability of evidence-based services that leads to more patients experiencing symptom decline and improvement of life.

Key practice points

(1) Pre-professionals can establish self-efficacy conducting CBT techniques following experiential training.

(2) Sensitivity to disgust may be an important clinician level variable to consider in the practice of exposure therapy.

(3) Providing early and enriched training experiences with CBT techniques may aid to improve dissemination.

Supplementary material. To view supplementary material for this article, please visit: https://doi.org/10.1017/ S1754470X22000010

Acknowledgements. None.

Author contributions. Ryan McCarty: Conceptualization (supporting), Data curation (equal), Formal analysis (lead), Investigation (equal), Methodology (supporting), Project administration (equal), Writing - original draft (lead), Writing review \& editing (lead); Danielle Cooke: Conceptualization (lead), Data curation (equal), Investigation (equal), Methodology (lead), Project administration (equal), Writing - review \& editing (supporting); Lacie Lazaroe: Conceptualization (supporting), Investigation (equal), Methodology (supporting), Project administration (supporting), Writing - original draft (supporting), Writing - review \& editing (supporting); Andrew Guzick: Conceptualization (supporting), Investigation (supporting), Methodology (supporting), Writing - review \& editing (supporting); Andrea Guastello: Supervision (supporting), Writing - original draft (supporting), Writing - review \& editing (supporting); Sierra Budd: Data curation (equal), Investigation (supporting), Project administration (equal), 
Writing - review \& editing (supporting); Seth Downing: Project administration (supporting), Writing - original draft (supporting), Writing - review \& editing (supporting); Ashley Ordway: Investigation (equal), Methodology (supporting), Project administration (supporting), Writing - review \& editing (supporting); Carol Mathews: Resources (lead), Supervision (supporting), Writing - review \& editing (supporting); Joseph McNamara: Conceptualization (supporting), Formal analysis (supporting), Investigation (supporting), Supervision (lead), Writing - original draft (supporting), Writing - review \& editing (supporting).

Financial support. This research did not receive any specific grant from funding agencies in the public, commercial, or notfor-profit sectors.

Conflicts of interest. The authors report no conflicts of interest.

Ethics statements. All authors have abided by the Ethical Principles of Psychologists and Code of Conduct as set out by the APA. This study was approved by the University of Florida Institutional Review Board (IRB201902582).

Data availability statement. The data that support the findings of this study are available from the corresponding author, R.M., upon reasonable request.

Further reading.

Balkhi, A. M., Reid, A. M., Guzick, A. G., Geffken, G. R., \& McNamara, J. P. H. (2016). The progress cascading model: a scalable model for teaching and mentoring graduate trainees in exposure therapy. Journal of Obsessive-Compulsive and Related Disorders, 9, 36-42. https://doi.org/10.1016/j.jocrd.2016.02.005

Beidas, R. S., \& Kendall, P. C. (2010). Training therapists in evidence-based practice: a critical review of studies from a systems-contextual perspective. Clinical Psychology: Science and Practice, 17, 1-30. https://doi.org/10.1111/j.1468-2850. 2009.01187.x

Deacon, B. J., Farrell, N. R., Kemp, J. J., Dixon, L. J., Sy, J. T., Zhang, A. R., \& McGrath, P. B. (2013). Assessing therapist reservations about exposure therapy for anxiety disorders: the Therapist Beliefs about Exposure Scale. Journal of Anxiety Disorders, 27, 772-780. https://doi.org/10.1016/j.janxdis.2013.04.006

Reid, A. M., Guzick, A. G., Balkhi, A. M., McBride, M., Geffken, G. R., \& McNamara, J. P. H. (2017). The progressive cascading model improves exposure delivery in trainee therapists learning exposure therapy for obsessive-compulsive disorder. Training and Education in Professional Psychology, 11, 260-265. https://doi.org/10.1037/tep0000159

\section{References}

Abramowitz, J. S., Deacon, B. J., \& Whiteside, S. P. H. (2019). Exposure Therapy for Anxiety Second Edition: Principles and Practice. Guilford Publications.

Becker, C. B., Zayfert, C., \& Anderson, E. (2004). A survey of psychologists' attitudes towards and utilization of exposure therapy for PTSD. Behaviour Research and Therapy, 42, 277-292. https://doi.org/10.1016/S0005-7967(03)00138-4

Broicher, T., Gerlach, A. L., \& Neudeck, P. (2017). Die Relevanz der Ausbildung für den späteren Einsatz von Expositionsverfahren in der therapeutischen Praxis. Zeitschrift Für Klinische Psychologie Und Psychotherapie, 46, 107-116. https://doi.org/10.1026/1616-3443/a000415

Callahan, J. L., \& Watkins Jr, C. E. (2018). The science of training II: prepracticum and practicum training. Training and Education in Professional Psychology, 12, 231-244. https://doi.org/10.1037/tep0000209

Choi, B. Y., Park, H., Yang, E., Lee, S. K., Lee, Y., \& Lee, S. M. (2012). Understanding career decision self-efficacy: a metaanalytic approach. Journal of Career Development, 39, 443-460. https://doi.org/10.1177/0894845311398042

Chu, B. C., Crocco, S. T., Arnold, C. C., Brown, R., Southam-Gerow, M. A., \& Weisz, J. R. (2015). Sustained implementation of cognitive-behavioral therapy for youth anxiety and depression: long-term effects of structured training and consultation on therapist practice in the field. Professional Psychology, Research and Practice, 46, 70-79. https://doi.org/10.1037/a0038000

Consedine, N. S., Yu, T.-C., \& Windsor, J. A. (2013). Nursing, pharmacy, or medicine? Disgust sensitivity predicts career interest among trainee health professionals. Advances in Health Sciences Education, 18, 997-1008. https://doi.org/10.1007/ s10459-012-9439-z

Cook, J. M., Schnurr, P. P., Biyanova, T., \& Coyne, J. C. (2009). Apples don't fall far from the tree: influences on psychotherapists' adoption and sustained use of new therapies. Psychiatric Services, 60, 671-676. https://doi.org/10. 1176/ps.2009.60.5.671

Deacon, B. J., Lickel, J. J., Farrell, N. R., Kemp, J. J., \& Hipol, L. J. (2013). Therapist perceptions and delivery of interoceptive exposure for panic disorder. Journal of Anxiety Disorders, 27, 259-264. https://doi.org/10.1016/j.janxdis. 2013.02.004

Deacon, B., \& Olatunji, B. O. (2007). Specificity of disgust sensitivity in the prediction of behavioral avoidance in contamination fear. Behaviour Research and Therapy, 45, 2110-2120. https://doi.org/10.1016/j.brat.2007.03.008 
Farrell, N. R., Deacon, B. J., Kemp, J. J., Dixon, L. J., \& Sy, J. T. (2013). Do negative beliefs about exposure therapy cause its suboptimal delivery? An experimental investigation. Journal of Anxiety Disorders, 27, 763-771. https://doi.org/10.1016/j. janxdis.2013.03.007

Farrell, N. R., Kemp, J. J., Blakey, S. M., Meyer, J. M., \& Deacon, B. J. (2016). Targeting clinician concerns about exposure therapy: a pilot study comparing standard vs. enhanced training. Behaviour Research and Therapy, 85, 53-59. https://doi. org/10.1016/j.brat.2016.08.011

Freiheit, S. R., Vye, C., Swan, R., \& Cady, M. (2004). Cognitive-behavioral therapy for anxiety: is dissemination working? The Behavior Therapist, 27, 25-32.

Gega, L., Norman, I. J., \& Marks, I. M. (2007). Computer-aided vs. tutor-delivered teaching of exposure therapy for phobia/ panic: randomized controlled trial with pre-registration nursing students. International Journal of Nursing Studies, 44, 397-405. https://doi.org/10.1016/j.ijnurstu.2006.02.009

Gunter, R. W., \& Whittal, M. L. (2010). Dissemination of cognitive-behavioral treatments for anxiety disorders: overcoming barriers and improving patient access. Clinical Psychology Review, 30, 194-202. https://doi.org/10.1016/j.cpr.2009.11.001

Hamerman, E. J., \& Schneider, A. B. (2018). The role of disgust sensitivity in volunteer recruitment and retention. International Journal of Nonprofit and Voluntary Sector Marketing, 23, e1597. https://doi.org/10.1002/nvsm.1597

Harned, M. S., Dimeff, L. A., Woodcock, E. A., \& Contreras, I. (2013). Predicting adoption of exposure therapy in a randomized controlled dissemination trial. Journal of Anxiety Disorders, 27, 754-762. https://doi.org/10.1016/j.janxdis. 2013.02.006

Harned, M. S., Dimeff, L. A., Woodcock, E. A., Kelly, T., Zavertnik, J., Contreras, I., \& Danner, S. M. (2014). Exposing clinicians to exposure: a randomized controlled dissemination trial of exposure therapy for anxiety disorders. Behavior Therapy, 45, 731-744. https://doi.org/10.1016/j.beth.2014.04.005

Harned, M. S., Dimeff, L. A., Woodcock, E. A., \& Skutch, J. M. (2011). Overcoming barriers to disseminating exposure therapies for anxiety disorders: a pilot randomized controlled trial of training methods. Journal of Anxiety Disorders, 25, 155-163. https://doi.org/10.1016/j.janxdis.2010.08.015

Higa-McMillan, C., Kotte, A., Jackson, D., \& Daleiden, E. L. (2017). Overlapping and non-overlapping practices in usual and evidence-based care for youth anxiety. Journal of Behavioral Health Services \& Research, 44, 684-694. https://doi.org/ 10.1007/s11414-016-9502-2

Hipol, L. J., \& Deacon, B. J. (2013). Dissemination of evidence-based practices for anxiety disorders in Wyoming: a survey of practicing psychotherapists. Behavior Modification, 37, 170-188. https://doi.org/10.1177/0145445512458794

Holstermann, N., Grube, D., \& Bögeholz, S. (2009). The influence of emotion on students' performance in dissection exercises. Journal of Biological Education, 43, 164-168. https://doi.org/10.1080/00219266.2009.9656177

Jacoby, R. J., Berman, N. C., Reese, H. E., Shin, J., Sprich, S., Szymanski, J., Pollard, C. A., \& Wilhelm, S. (2019). Disseminating cognitive-behavioral therapy for obsessive compulsive disorder: comparing in person vs. online training modalities. Journal of Obsessive-Compulsive and Related Disorders, 23, 100485. https://doi.org/10.1016/j.jocrd.2019.100485

Klepac, R. K., Ronan, G. F., Andrasik, F., Arnold, K. D., Belar, C. D., Berry, S. L., Christofff, K. A., Craighead, L. W., Dougher, M. J., Dowd, E. T., Herbert, J. D., McFarr, L. M., Rizvi, S. L., Sauer, E. M., \& Strauman, T. J. (2012). Guidelines for cognitive behavioral training within doctoral psychology programs in the United States: report of the Inter-Organizational Task Force on Cognitive and Behavioral Psychology Doctoral Education. Behavior Therapy, 43, 687-697. https://doi.org/10.1016/j.beth.2012.05.002

Levita, L., Salas Duhne, P. G., Girling, C., \& Waller, G. (2016). Facets of clinicians' anxiety and the delivery of cognitive behavioral therapy. Behaviour Research and Therapy, 77, 157-161. https://doi.org/10.1016/j.brat.2015.12.015

McDonough, M., \& Marks, I. M. (2002). Teaching medical students exposure therapy for phobia/panic - randomized, controlled comparison of face-to-face tutorial in small groups vs. solo computer instruction. Medical Education, 36, 412-417. https://doi.org/10.1046/j.1365-2923.2002.01210.x

McGuire, J. F., Wu, M. S., Choy, C., \& Piacentini, J. (2018). Editorial Perspective: Exposures in cognitive behavior therapy for pediatric obsessive-compulsive disorder: addressing common clinician concerns. Journal of Child Psychology and Psychiatry, and Allied Disciplines, 59, 714-716. https://doi.org/10.1111/jcpp.12818

Meyer, J. M., Farrell, N. R., Kemp, J. J., Blakey, S. M., \& Deacon, B. J. (2014). Why do clinicians exclude anxious clients from exposure therapy? Behaviour Research and Therapy, 54, 49-53. https://doi.org/10.1016/j.brat.2014.01.004

Olatunji, B. O., Armstrong, T., \& Elwood, L. (2017). Is disgust proneness associated with anxiety and related disorders? A qualitative review and meta-analysis of group comparison and correlational studies. Perspectives on Psychological Science, 12, 613-648. https://doi.org/10.1177/1745691616688879

Olatunji, B. O., Deacon, B. J., \& Abramowitz, J. S. (2009). The cruelest cure? Ethical issues in the implementation of exposure-based treatments. Cognitive and Behavioral Practice, 16, 172-180. https://doi.org/10.1016/j.cbpra.2008.07.003

Olatunji, B. O., Williams, N. L., Tolin, D. F., Abramowitz, J. S., Sawchuk, C. N., Lohr, J. M., \& Elwood, L. S. (2007). The Disgust Scale: item analysis, factor structure, and suggestions for refinement. Psychological Assessment, 19, 281-297. https://doi.org/10.1037/1040-3590.19.3.281

Pittig, A., \& Hoyer, J. (2017). Exposition aus Sicht niedergelassener Verhaltenstherapeutinnen und Verhaltenstherapeuten: Anwendung und systemische Barrieren. [Utilization and barriers of exposure in private practice: the perspective of 
behavioral psychotherapists.]. Zeitschrift Für Klinische Psychologie und Psychotherapie: Forschung und Praxis, 46, 223-235. https://doi.org/10.1026/1616-3443/a000441

Pittig, A., Kotter, R., \& Hoyer, J. (2019). The struggle of behavioral therapists with exposure: self-reported practicability, negative beliefs, and therapist distress about exposure-based interventions. Behavior Therapy, 50, 353-366. https://doi. org/10.1016/j.beth.2018.07.003

Randler, C., Hummel, E., \& Wüst-Ackermann, P. (2013). The influence of perceived disgust on students' motivation and achievement. International Journal of Science Education, 35, 2839-2856. https://doi.org/10.1080/09500693.2012. 654518sarssa

Reese, H. E., Pollard, C. A., Szymanski, J., Berman, N., Crowe, K., Rosenfield, E., \& Wilhelm, S. (2016). The Behavior Therapy Training Institute for OCD: a preliminary report. Journal of Obsessive-Compulsive and Related Disorders, 8, 79-85. https://doi.org/10.1016/j.jocrd.2015.12.005

Reid, A. M., Bolshakova, M. I., Guzick, A. G., Fernandez, A. G., Striley, C. W., Geffken, G. R., \& McNamara, J. P. (2017). Common barriers to the dissemination of exposure therapy for youth with anxiety disorders. Community Mental Health Journal, 53, 432-437. https://doi.org/10.1007/s10597-017-0108-9

Reid, A. M., Guzick, A. G., Fernandez, A. G., Deacon, B., McNamara, J. P. H., Geffken, G. R., McCarty, R., \& Striley, C. W. (2018). Exposure therapy for youth with anxiety: utilization rates and predictors of implementation in a sample of practicing clinicians from across the United States. Journal of Anxiety Disorders, 58, 8-17. https://doi.org/10. 1016/j.janxdis.2018.06.002

Sars, D., \& van Minnen, A. (2015). On the use of exposure therapy in the treatment of anxiety disorders: a survey among cognitive behavioural therapists in the Netherlands. BMC Psychology, 3, 26. https://doi.org/10.1186/s40359-015-0083-2

Scherr, S. R., Herbert, J. D., \& Forman, E. M. (2015). The role of therapist experiential avoidance in predicting therapist preference for exposure treatment for OCD. Journal of Contextual Behavioral Science, 4, 21-29. https://doi.org/10.1016/j. jcbs.2014.12.002

Schumacher, S., Gaudlitz, K., Plag, J., Miller, R., Kirschbaum, C., Fehm, L., Fydrich, T., \& Ströhle, A. (2014). Who is stressed? A pilot study of salivary cortisol and alpha-amylase concentrations in agoraphobic patients and their novice therapists undergoing in vivo exposure. Psychoneuroendocrinology, 49, 280-289. https://doi.org/10.1016/j.psyneuen. 2014.07.016

Smits, J. A. J., Telch, M. J., \& Randall, P. K. (2002). An examination of the decline in fear and disgust during exposure-based treatment. Behaviour Research and Therapy, 40, 1243-1253. https://doi.org/10.1016/S0005-7967(01)00094-8

Turner, K. M. T., Nicholson, J. M., \& Sanders, M. R. (2011). The role of practitioner self-efficacy, training, program and workplace factors on the implementation of an evidence-based parenting intervention in primary care. Journal of Primary Prevention, 32, 95-112. https://doi.org/10.1007/s10935-011-0240-1

van Minnen, A., Hendriks, L., \& Olff, M. (2010). When do trauma experts choose exposure therapy for PTSD patients? A controlled study of therapist and patient factors. Behaviour Research and Therapy, 48, 312-320. https://doi.org/10. 1016/j.brat.2009.12.003

Waller, G., \& Turner, H. (2016). Therapist drift redux: why well-meaning clinicians fail to deliver evidence-based therapy, and how to get back on track. Behaviour Research and Therapy, 77, 129-137. https://doi.org/10.1016/j.brat.2015.12.005

Whiteside, S. P. H., Deacon, B. J., Benito, K., \& Stewart, E. (2016). Factors associated with practitioners' use of exposure therapy for childhood anxiety disorders. Journal of Anxiety Disorders, 40, 29-36. https://doi.org/10.1016/j.janxdis.2016.04. 001

Whiteside, S. P. H., Sattler, A., Ale, C. M., Young, B., Hillson Jensen, A., Gregg, M. S., \& Geske, J. R. (2016). The use of exposure therapy for child anxiety disorders in a medical center. Professional Psychology: Research and Practice, 47, 206-214. https://doi.org/10.1037/pro0000077

Zoellner, L. A., Feeny, N. C., Bittinger, J. N., Bedard-Gilligan, M. A., Slagle, D. M., Post, L. M., \& Chen, J. A. (2011). Teaching trauma-focused exposure therapy for PTSD: critical clinical lessons for novice exposure therapists. Psychological Trauma: Theory, Research, Practice and Policy, 3, 300-308. https://doi.org/10.1037/a0024642

Cite this article: McCarty RJ, Cooke DL, Lazaroe LM, Guzick AG, Guastello AD, Budd SM, Downing ST, Ordway AR, Mathews CA, and McNamara JPH. The effects of an exposure therapy training program for pre-professionals in an intensive exposure-based summer camp. The Cognitive Behaviour Therapist. https://doi.org/10.1017/S1754470X22000010 